\title{
A NOTE ON A THEOREM OF $K$. G. WOLFSON
}

\section{J. HEIDER ${ }^{1}$}

1. In [3] K. G. Wolfson proves the following:

Theorem. $A$ commutative $B^{*}$-algebra $K$ containing an identity $e$ (with $\left\|k^{*} \cdot k\right\|=\|k\|^{2}$ for all $k \in K$ and $\|e\|=1$ ) is isomorphic (in a norm and * preserving manner) to an algebra $B(X)$ of all bounded complexvalued functions on an essentially unique set $X$ if and only if:

(1) Every nonzero closed ideal of $K$ contains a minimal ideal;

(2) The sum of two annulets is an annulet.

An annulet is here understood to be an ideal $I$ of $K$ with which is associated a subset $G \subseteq K$ such that

$$
I=[k \in K \mid k \cdot g=0 \text { for all } g \in G] \text {. }
$$

The two characterizing traits therein presented neither are selfevident in $B(X)$ nor touch upon the projections and Gelfand-Neumark $[1 ; 2]$ continuous function representations so commonly used in the analysis of $B^{*}$-algebras. It is with this in mind that the following characterizations are offered:

ThEOREM 1. A necessary and sufficient condition that a $B^{*}$-algebra $C(X)$ of all continuous complex-valued functions on a compact Hausdorff space $X$ be isomorphic (in a norm and * preserving manner) to a $B^{*}$-algebra $B\left(\bar{X}_{0}\right)$ of all bounded complex-valued functions on a set $\bar{X}_{0}$ (essentially unique) is that the compact space $X$ contain a dense subset $X_{0}$ of points each of which is an open-closed subset of $X$ and such that each nonempty subset of points in $X_{0}$ is contained in an open-closed subset of $X$ which includes of $X_{0}$ just that subset.

Proof. Sufficiency. Assume that the compact Hausdorff space $X$ contains a subset $X_{0}$ as described above. Viewing $X_{0}$ simply as a set, form the $B^{*}$-algebra $B\left(X_{0}\right)$ and consider the map $C(X) \rightarrow B\left(X_{0}\right)$ under which $c$ in $C(X)$ becomes $\bar{c}$ in $B\left(X_{0}\right)$ with $\bar{c}\left(x_{0}\right)=c\left(x_{0}\right)$ for all $x_{0}$ in $X_{0}$. This map is linear and ${ }^{*}$ preserving. Since $X_{0}$ is dense in $X$ the map is norm preserving and thus $1-1$. Finally this map is onto: thus let $b \in B\left(X_{0}\right)$ be arbitrary and assume that its range as $x_{0}$ varies over $X_{0}$ falls in the interior of a right-open square of side $2 M$ about the origin in the complex plane. For each positive integer $n$ the de-

Received by the editors June 24, 1954. igan.

1 The author is a Fellow of the Rackham Foundation of the University of Mich- 
composition of this square into $2^{n} \times 2^{n}$ equal right-open squares determines a division of $X_{0}$ into $2^{n} \times 2^{n}$ disjoint subsets (some perhaps empty) and thus a decomposition of $X$ into $2^{n} \times 2^{n}$ disjoint openclosed subsets (some perhaps the empty set) $X_{i}, i=1, \cdots, 2^{n} \times 2^{n}$. If $\lambda_{i}$ is the center of the $i$ th of the above squares and $\chi\left(X_{i}\right)$ is the characteristic function of the corresponding open-closed subset of $X$, then $c_{n}(x)=\sum_{i=1}^{2^{n} \times 2^{n}} \lambda_{i} \cdot \chi\left(X_{i}\right)[x]$ is an element of $C(X)$ such that $\mid \bar{c}_{n}\left(x_{0}\right)$ $-b\left(x_{0}\right) \mid \leqq M / n 2^{1 / 2}$ for all $x_{0}$ in $X_{0}$. Thus $\left\{\bar{c}_{n}\right\}$ is a Cauchy sequence in $B\left(X_{0}\right)$ and, since norms are preserved, $\left\{c_{n}\right\}$ is a Cauchy sequence in $C(X)$ which converges point-wise (and uniformly) to an element $c \in C(X)$. Finally, for each $x_{0}$ in $X_{0}$ :

$$
c\left(x_{0}\right)=\lim _{n} c_{n}\left(x_{0}\right)=\lim _{n} \bar{c}_{n}\left(x_{0}\right)=b\left(x_{0}\right) .
$$

Necessity. Assume now that $C(X)$ is isomorphic as a $B^{*}$-algebra to some $B\left(\bar{X}_{0}\right)$. In $B\left(\bar{X}_{0}\right)$ denote by $\bar{e}_{\bar{x}_{0}}, \bar{x}_{0} \in \bar{X}_{0}$, the system of all characteristic functions of a single point $\bar{x}_{0} \in \bar{X}_{0}$ and by $\bar{e}_{\bar{A}}, \bar{A} \in S\left(\bar{X}_{0}\right)$, the system of all characteristic functions on the various subsets $\bar{A} \in S\left(\bar{X}_{0}\right)$ of $\bar{X}_{0}$. Denote by $e_{x_{0}}$ and $e_{\bar{A}}$ the elements of $C(X)$ which correspond under the assumed isomorphism to $\bar{e}_{\bar{x}_{0}}$ and $\bar{e}_{\bar{A}}$ in $B\left(\bar{X}_{0}\right)$. It is clear that $e_{x_{0}}$ and $e_{A}^{\prime \prime \prime}$ are idempotent, hermitian in $C(X)$ and thus are the characteristic functions of open-closed subsets of $X$. For each $c \in C(X)$ and each $e_{\bar{x}_{0}}, c \cdot e_{\bar{x}_{0}}=\lambda e_{x_{0}}$ where $\lambda$ is a complex scalar, since the corresponding multiplicative property clearly holds in $B\left(\bar{X}_{0}\right)$. From this it follows, by use of the theorem that for any open set $O$ in compact $X$ and any point $x_{0}$ in $O$ there is an element $c$ in $C(X)$ with $c\left(x_{0}\right)=1$ and $c(x)=0$ for $x \in O$, that $e_{x_{0}}$ is the characteristic function of a single point $x_{0} \in X$. Similarly for each $c \neq 0$ in $C(X)$ there exists at least one $e_{\bar{x}_{0}}$ in $C(X)$ such that $c \cdot e_{\bar{x}_{0}} \neq 0$ since the corresponding property holds in $B\left(\bar{X}_{0}\right)$. From this it follows by the same theorem that the points $x_{0}$ determined by the $e_{\tilde{x}_{0}}$ are dense in $X$. Finally for any collection of the $x_{0}$ in $X$ the open-closed subset $A$ of $X$ determined by the idempotent, hermitian element $e_{\bar{A}}$ of $C(X)$ corresponding to the element $\bar{e}_{\bar{A}}$ of $B\left(\bar{X}_{0}\right)$, where $\bar{A}$ contains all and only the corresponding $\bar{x}_{0}$, itself contains all and only the given $x_{0}$ since $e_{\bar{x}_{0}} \cdot e_{\bar{A}}=e_{\bar{x}_{0}}$ if and only if $\bar{e}_{\bar{x}_{0}} \cdot \bar{e}_{\bar{A}}=\bar{e}_{\bar{x}_{0}}$.

Since a dense subset in $X$ of points that are open-closed subsets necessarily includes all such points, the essential identity of the sets $\bar{X}_{0}, X_{0}$ is clear.

2. Let $K$ be an arbitrary commutative $B^{*}$-algebra with unit $e$, $\|e\|=1$, and with $\left\|k^{*} \cdot k\right\|=\|k\|^{2}$ for all $k \in K$. In the collection of all projections (idempotent, hermitian, nonzero elements) of $K$ distin- 
guish the collection (possibly empty) $e_{x_{0}}, x_{0} \in X_{0}$, of projections such that for each $k \in K$ and each $e_{x_{0}}, x_{0} \in X_{0}$, there exists a complex scalar $\lambda$ such that $k \cdot e_{x_{0}}=\lambda e_{x_{0}}$. Such projections may be called minimal projections. Here the indexing set $X_{0}$ is assumed so chosen that $x_{0} \neq y_{0}$ in $X_{0}$ implies $e_{x_{0}} \neq e_{y_{0}}$ in $K$.

THEOREM 2. A necessary and sufficient condition that a commutative $B^{*}$-algebra $K$ with identity $e$ and with $\left\|k^{*} \cdot k\right\|=\|k\|^{2}$ for all $k$ be isomorphic (in a norm and * preserving manner) to a $B^{*}$-algebra $B\left(\bar{X}_{0}\right)$ of all complex-valued functions on a set $\bar{X}_{0}$ is that the subset of all minimal projections $e_{x_{0}}, x_{0} \in X_{0}$, of $K$ be such that:

(1) for each $k \neq 0$ in $K$ there exists at least one minimal projection $e_{x_{0}}, x_{0} \in X_{0}$, such that $k \cdot e_{x_{0}} \neq 0$.

(2) for each subcollection $A \subseteq X_{0}$ of minimal projections there exists in $K$ a projection $e_{A}$ such that $e_{A} \cdot e_{x_{0}}=e_{x_{0}}$ for $x_{0} \in A$ and $e_{A} \cdot e_{x_{0}}=0$ for $x_{0} \notin A$.

When these conditions are satisfied the indexing set $X_{0}$ may be identified with the set $\bar{X}_{0}$.

Proof. Necessity. Assume $K$ isomorphic to $B\left(\bar{X}_{0}\right)$. It is then evident that the minimal projections $e_{x_{0}}, x_{0} \in X_{0}$, of $K$ correspond in a $1-1$, onto manner to the characteristic functions in $B\left(\bar{X}_{0}\right)$ of single points $\bar{x}_{0}$ of $\bar{X}_{0}$, that the indexing set $X_{0}$ may be identified with the set $\bar{X}_{0}$, and that the two conditions given above are satisfied with the $e_{A}$ of condition (2) being the inverse images in $K$ of the characteristic functions in $B\left(\bar{X}_{0}\right)$ of various subsets $\bar{A}_{0}$ of $\bar{X}_{0}$.

Sufficiency. Assume that the collection (now nonempty) $e_{x_{0}}, x_{0} \in X_{0}$, of minimal projections in $K$ satisfies the stated conditions. By the Gelfand-Neumark theory $[1 ; 2] K$ is isomorphic (in a norm and * preserving manner) to an algebra $C(\bar{X})$ of all continuous complexvalued functions on a compact Hausdorff space $\bar{X}$. Let $\bar{X}_{0}$ denote the set of all points $\bar{x}_{0}$ which are open-closed sets in $\bar{X}$. Using again the theorem that for each point $\bar{y}$ in an open set $O$ of $\bar{X}$ there exists an element $c$ of $C(\bar{X})$ with $c(\bar{y})=1$ and $c(\bar{x})=0$ for $\bar{x} \notin O$, it is clear that the minimal projections $e_{x_{0}}, x_{0} \in X_{0}$, of $K$ correspond in a 1-1 onto manner to the collection $e_{\bar{x}_{0}}, \bar{x}_{0} \in \bar{X}_{0}$, of all characteristic functions on single point, open-closed sets of $\bar{X}$, that $X_{0}$ and $\bar{X}_{0}$ may be identified, that (by condition 1) the points of $\bar{X}_{0}$ are dense in $\bar{X}$, and that (by condition (2)) each nonempty subset of points in $\bar{X}_{0}$ is contained in an open-closed subset of $\bar{X}$ which contains all and only those points of $\bar{X}_{0}$ that are in the given subset. It follows then by Theorem 1 that $C(\bar{X})$ and hence $K$ is isomorphic to a $B\left(X_{0}^{*}\right)$ wherein the set $X_{0}^{*}$ may be identified with $\bar{X}_{0}$ and thus with $X_{0}$. 


\section{REFERENCES}

1. I. Gelfand, Normierte Ringe, Rec. Math. (Mat. Sbornik) N. S. vol. 9 (1941) pp. 3-24.

2. I. Gelfand and M. Neumark, On the embedding of normed rings into the ring of operators in Hilbert space, Rec. Math. (Mat. Sbornik) N. S. vol. 12 (1943) pp. 197-213.

3. K. G. Wolfson, The algebra of bounded functions, Proc. Amer. Math. Soc. vol. 5 (1954) pp. 10-14.

UNIVERSity OF MichigaN

\section{A NOTE ON UNSTABLE HOMEOMORPHISMS ${ }^{1}$}

ROBERT F. WILLIAMS

In [1] W. R. Utz introduced the concept of an unstable homeomorphism and raised the question of whether there exists an unstable homeomorphism of a compact continuum onto itself. In this note an example of such an homeomorphism will be given.

Let $C$ denote the complex unit circle and for each $z \in C$, let $g(z)=z^{2}$. Then $g: C$ onto $C$ determines an inverse limit space $\Sigma_{2}$ $=\left\{\left(a_{0}, a_{1}, a_{2}, \cdots\right) \mid\right.$ for each non-negative integer $i, a_{i} \in C$ and $\left.g\left(a_{i+1}\right)=a_{i}\right\}$. For $a, b \in \Sigma_{2}$, the function $\rho(a, b)=\sum_{i=0}^{\infty}\left|a_{i}-b_{i}\right| / 2^{i}$ is a metric for $\Sigma_{2} ; \Sigma_{2}$ is familiar as the "two-solenoid," and is a compact, indecomposable continuum. Define $f: \Sigma_{2}$ onto $\Sigma_{2}$ as follows: for each $a=\left(a_{0}, a_{1}, \cdots\right) \in \Sigma_{2}$, let $f(a)=\left[g\left(a_{0}\right), g\left(a_{1}\right), \cdots\right]$. Then $f(a)$ $=\left(a_{0}^{2}, a_{1}^{2}, \cdots\right)=\left(a_{0}^{2}, a_{0}, a_{1}, \cdots\right), f^{-1}(a)=\left(a_{1}, a_{2}, a_{3}, \cdots\right)$, and $f$ is a homeomorphism of $\Sigma_{2}$ onto $\Sigma_{2}$.

To show that $f$ is unstable, suppose that $a=\left(a_{0}, a_{1}, \cdots\right)$ and $b=\left(b_{0}, b_{1}, \cdots\right)$ are distinct points of $\Sigma_{2}$. Consider, as Case 1 , that $a_{0} \neq b_{0}$. Let $e^{i \theta}=a_{0}, e^{i \phi}=b_{0}$, where $0 \leqq \theta, \phi<2 \pi$. Then there exists a non-negative integer $n$ such that the angle between the terminal rays of $2^{n} \theta$ and $2^{n} \phi$ is greater than $\pi / 2$. Then $\rho\left[f^{n}(a), f^{n}(b)\right] \geqq\left|a_{0}^{2^{n}}-b_{0}^{2^{n}}\right|$ $=\left|e^{i 2^{n} \theta}-e^{i 2^{n} \phi}\right|>1$.

Case 2: for some integer $n>0, a_{n} \neq b_{n}$, but $a_{i}=b_{i}$, for $0 \leqq i<n$. Then $f^{-n}(a)=\left(a_{n}, a_{n+1}, a_{n+2}, \cdots\right), f^{-n}(b)=\left(b_{n}, b_{n+1}, b_{n+2}, \cdots\right)$, and there-

Presented to the Society, November 26, 1954; received by the editors June 25, 1954.

1 Research under NSF-G358, National Science Foundation.

${ }^{2}$ A homeomorphism $f$ of a compact metric space $X$ onto $X$ is said to be unstable provided there exists a fixed positive number $\delta$, such that if $x$ and $y$ are distinct points of $X$, then there exists an integer $n$, such that $\rho\left[f^{n}(x), f^{n}(y)\right]$ is greater than $\delta$. 